\title{
Hypersensitivity reactions to non-steroidal anti- inflammatory drugs: results of an Austrian cohort study
}

\author{
Teresa Bangerl · Brigitte Zahel · Andrea Lueger · Emmanuella Guenova - Irena Angelova-Fischer • \\ Wolfram Hoetzenecker
}

Received: 15 April 2020 / Accepted: 12 June 2020 / Published online: 14 July 2020

(C) The Author(s) 2020

\begin{abstract}
Summary
Background Hypersensitivity to non-steroidal antiinflammatory drugs (NSAIDs) is the second most common cause of drug hypersensitivity. Despite the importance of NSAIDs in routine analgesia only few studies have systematically addressed the question of tolerability in hypersensitive patients.

Methods The authors retrospectively analysed 398 patients that were treated at the Department of Dermatology, Kepler University Hospital Linz, Austria, in the period 2012-2016 with a clinical history of NSAID hypersensitivity. Skin tests (skin prick and intracutaneous tests) to common NSAIDs were performed, followed by single-blinded, placebo-controlled drug challenge with either the culprit drug or an alternative NSAID.

Results A total of 361 patients were subjected to skin testing. Of these, 25 patients $(6.3 \%)$ showed a positive reaction to the culprit drug. According to the severity of the reaction in the medical history, 87 patients were exposed orally to the culprit drug (oral provocation test, OPT) after negative skin test and 255 patients received OPT with alternative NSAIDs ac-
\end{abstract}

Availability of data and materials The datasets generated during and/or analysed during the current study are available from the corresponding author on reasonable request.

T. Bangerl · B. Zahel · A. Lueger · I. Angelova-Fischer · W. Hoetzenecker $(\varangle)$

Department of Dermatology, Kepler University Hospital, Johannes Kepler University Linz, Linz, Austria wolfram.hoetzenecker@kepleruniklinikum.at

\section{E. Guenova}

Department of Dermatology, University Hospital Zurich, Zurich, Switzerland

Department of Dermatology, University Hospital Lausanne, Lausanne, Switzerland cording to established protocols. OPT with the culprit drug resulted in hypersensitivity reactions in 12 patients $(13.79 \%)$. In terms of alternative NSAID testing, the three most commonly tested drugs were lornoxicam (192 OPTs), acetaminophen (156 OPTs) and celecoxib (133 OPTs) with tolerability rates in respectively 88.54\% (hypersensitivity reactions, $11.46 \%$ ), $92.31 \%$ (hypersensitivity reactions, $7.69 \%$ ) and $91.73 \%$ (hypersensitivity reactions, $8.27 \%$ ) of cases.

Conclusion OPT with alternative NSAIDs are useful in patients with NSAID hypersensitivity as tolerability varies between the individual substances.

Keywords NSAID · Hypersensitivity · Alternative drugs $\cdot$ Drug reaction · Allergy

$\begin{array}{ll}\text { Abbreviations } \\ \text { ASA } & \text { Acetylsalicylic acid } \\ \text { ICT } & \text { Intracutaneous test } \\ \text { NSAID } & \text { Non-steroidal anti-inflammatory drug } \\ \text { OPT } & \text { Oral provocation test } \\ \text { SPT } & \text { Skin prick test } \\ \text { ST } & \text { Skin test }\end{array}$

\section{Introduction}

Hypersensitivity reactions to non-steroidal anti-inflammatory drugs (NSAID) comprise a broad spectrum of clinical and organ manifestations and have been considered the second most common cause of drug hypersensitivity [1]. Based on published epidemiological data, aspirin hypersensitivity alone may affect $0.5 \%-1.9 \%$ of the general population, whereas the estimated incidence of NSAID hypersensitivity in the same group ranges from 0.6 to $2.5 \%$ [2-4]. Depending on the data source, the incidence of NSAID hypersensitivity in individuals with bronchial asthma may reach $5-10 \%$ and increase further in 
the presence of concomitant asthma, rhinosinusitis and nasal polyps or underlying chronic urticaria [5, 6]. The power of COX-1 inhibitors is directly related to the cross-reactivity between NSAIDs in patients with NSAID-exacerbated respiratory disease (NERD). COX-2 inhibitors are mostly well tolerated by these patients [7].

NSAID hypersensitivity reactions vary in terms of symptoms, time of onset, underlying chronic diseases and the involved pathophysiologic mechanisms or biochemical pathways [8-10]. The commonly used classification system of NSAID intolerance comprises one respiratory type (NERD), two skin types (NSAIDinduced urticaria/angioedema [NIUA/NSAID] exacerbated cutaneous disease [NECD]) and two allergic types (single NSAID-induced urticara/angioedema or anaphylaxis [SNIUAA] and single NSAID-induced delayed reaction [SNIDR]) [11-13].

As most reactions relate to non-IgE mediated mechanisms, despite the clinical importance and performed research, there are no reliable skin or laboratory tests as yet for the accurate diagnosis of NSAID hypersensitivity. In this context, provocation tests remain the 'gold standard' to identify the suspected causative agent and subsequently confirm or exclude the diagnosis of NSAID hypersensitivity. Furthermore, provocation tests allow the identification of alternative compounds in patients with previous NSAID reactions [14-17].

The present study analysed data from 398 patients with NSAID hypersensitivity in their medical history with the aim to: (i) identify the causative agents for the reported reactions to NSAIDs and (ii) gain information on the tolerability of the alternative, skin test-negative NSAIDs administered in an oral provocation challenge test.

\section{Material and methods}

\section{Patients}

The authors included 398 patients with a clinical history of NSAID hypersensitivity from 2012 to 2016, retrospectively. The patients were registered in the database of the Department of Dermatology, Kepler University Hospital Linz, Austria. All patients with a clinical history of NSAID hypersensitivity (e.g. urticaria, flush, gastrointestinal symptoms, anaphylactic reactions, angioedema, bronchial asthma, nasal polyps) were included in the study. Serum tryptase and total immunoglobulin E (IgE) were assessed in all patients. The recorded data included demography, culprit drugs in the medical history, the clinical manifestations of the hypersensitivity reactions, additional allergies and trigger factors (e.g. bronchial asthma, chronic urticaria/angioedema).

\section{Drugs}

The drugs administered in oral provocation testing (OPT) were relevant to the Austrian market. This reflects the correspondence to the market and prescription practices in Austria and may not be marketed in other European countries. The same refers to the frequencies of prescriptions due to the drug (country-specific) regulations. Others, such as propyphenazone, are no longer available on the market, but might still be of relevance to patients that have been previously exposed and sensitized to the drug. The selection of the specific NSAIDs for alternative OPT was based on the absolute/relative COX2 inhibition (e.g. celecoxib, Iornoxicam) of the drug and the lower upper provocation dose with a weaker NSAID (e.g. mefenamic acid, acetaminophen).

\section{Skin test $(S T)=$ skin prick test $(S P T)$ and intracutaneous test (ICT)}

Before oral provocation, all patients underwent a skin test (ST) according to standard protocols [18]. This was part of the routine workflow. Briefly, the skin prick test (SPT) and intracutaneous test (ICT) with a standard panel of NSAIDs, including the culprit drug, were performed on the forearm. The standard panel included: acetaminophen also known as paracetamol (SPT $10 \mathrm{mg} / \mathrm{ml} ;$ ICT $10 \mathrm{mg} / \mathrm{ml} \mathrm{1:10,} \mathrm{1:100,} \mathrm{1:1000),}$ mefenamic acid (SPT $100 \mathrm{mg} / \mathrm{ml}$ ), metamizole (SPT $40 \mathrm{mg} / \mathrm{ml}, 400 \mathrm{mg} / \mathrm{ml}$; ICT $0.4 \mathrm{mg} / \mathrm{ml}, 4 \mathrm{mg} / \mathrm{ml}, 40 \mathrm{mg} /$ ml) [19], prophyphenazone (SPT 0.16\%, 20\%), acetylsalicylic acid (ASA) (SPT $10 \mathrm{mg} / \mathrm{ml}$ ), diclofenac (SPT $25 \mathrm{mg}$, ICT $0.1 \mathrm{mg} / \mathrm{ml}, 1 \mathrm{mg} / \mathrm{ml}$ ), ibuprofen (SPT $100 \mathrm{mg} / \mathrm{ml}$ ), meloxicam (SPT $15 \mathrm{mg} / \mathrm{ml}$ ), lornoxicam (SPT $8 \mathrm{mg} / \mathrm{ml}$ ), indomethacin (SPT $50 \mathrm{mg} / \mathrm{ml}$ ) and celecoxib (SPT $100 \mathrm{mg} / \mathrm{ml}$ ) dissolved in sodium chloride. An ST with sodium chloride $0.9 \%$ and histamine $(10 \mathrm{mg} / \mathrm{ml})$ served negative and positive controls. SPT was considered positive if a weal and flare reaction appeared after $20 \mathrm{~min}$, in which the wheal was $3 \mathrm{~mm}$ larger than that of the negative control. ICT was considered positive if after $20 \mathrm{~min}$ the induced weal reaction was more than $3 \mathrm{~mm}$ larger than the saline control and larger than the original vesicle. OPT was only performed after negative skin testing with the NSAID standard panel.

\section{Oral provocation test}

OPT was performed as a single-blinded, placebocontrolled drug challenge. It was performed under continuous monitoring and all patients provided signed informed consent beforehand. Before starting, a dermatologic and general medical examination was performed. Patients with medical pre-conditions (e.g. uncontrolled bronchial asthma, severe heart disease), pregnancy, use of $\beta$-blockers and a medical history of severe cutaneous drug eruption were excluded from 
Table 1 Characteristics of the study cohort (number of patients, skin tests and provocation challenges)

\begin{tabular}{llll} 
& Total & ST & OPT \\
Patients $(n)$ & 398 & 361 & 342 \\
\hline Sex & & & \\
Female & $255(64.1 \%)$ & $235(65.1 \%)$ & $223(65.2 \%)$ \\
\hline Male & $143(35.9 \%)$ & $126(34.9 \%)$ & $119(34.8 \%)$ \\
\hline Age \pm SD (years) & $47.2 \pm 16.0$ & $46.7 \pm 16$ & $47.4 \pm 15.8$ \\
\hline \multicolumn{4}{ll}{ ST skin test, OPT oral provocation test, $S D$ standard deviation }
\end{tabular}

OPT. The order and number of the tested drugs was defined individually for each patient; the culprit drug was never administered on the first day. Placebo (either lactose or corn starch) was administered first, followed by individual NSAIDs, which were taken by the patient in increasing order with 60 -min intervals. Both the placebo and the individual NSAIDs were concealed in gelatine capsules and only one NSAID was tested each day. If a patient developed objective signs of an allergic reaction (e.g. flush, generalized pruritus, dyspnea, cough), the OPT was stopped immediately and the hypersensitivity reaction was regarded as positive. In the case of subjective symptoms (e.g. headache, localized pruritus, dizziness), the OPT was suspended for $2 \mathrm{~h}$ and continued after recovery. If subjective symptoms did not improve, the OPT was cancelled. The patients remained under surveillance for $24 \mathrm{~h}$ after the last challenge. Allergic reactions were graded according to Müller [20]. The administered cumulative dose of the individual NSAIDs were as follows: acetaminophen-850 mg, ASA $-810 \mathrm{mg}$, diclofenac- $187.5 \mathrm{mg}$, ibuprofen-650 mg, naproxen$550 \mathrm{mg}$, celecoxib-300 mg, lornoxicam-14 mg, mefenamic acid $-875 \mathrm{mg}$, metamizole $-425 \mathrm{mg}$, indomethacin $-50 \mathrm{mg}$, meloxicam- $15 \mathrm{mg}$.

\section{Results}

A total of 398 patients with a medical history of NSAID hypersensitivity were included in this study. Patient characteristics, test procedures and a flow chart are described in Table 1 and Fig. 1.

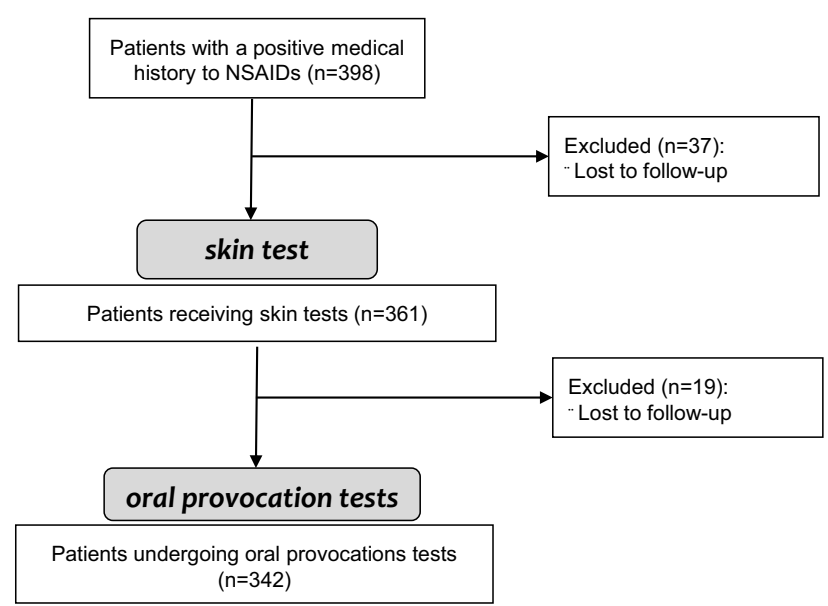

Fig. 1 Flow chart of the study. A total of 398 patients with a medical history of reactions to non-steroidal antiinflammatory drugs (NSAIDs) were included in this study. Of those, 361 patients were subjected to skin testing and 342 patients underwent oral provocation tests

Patients most commonly reported reactions to diclofenac (153 patients, 38.4\%), ASA (121 patients, $30.4 \%$ ), acetaminophen (106 patients, $26.6 \%$ ), ibuprofen/dexibuprofen (96 patients, 24.1\%), mefenamic acid (80 patients, $20.1 \%$ ) and metamizole (68 patients, $17.1 \%$ ) (Fig. 2). As part of the allergy work-up, the authors first performed ST (SPT and IC), including acetaminophen, mefenamic acid, metamizole (prophyphenazone), ASA, diclofenac or ibuprofen in 361 patients $(90.7 \%$; 37 patients did not show up for skin testing, see Fig. 1).

Interestingly, 25 patients $(6.3 \%)$ displayed a positive ST to the culprit drug, whereas 41 patients showed positive reactions in ST without any medical history (Tables 2 and 3). Next, 87 patients were subjected to single-blinded, placebo-controlled OPTs with the culprit drug after negative ST. In the case of severe reactions in the medical history (>grade I), no OPT was performed with the culprit drug and patients were subjected to testing with alternative NSAIDs $(n=255)$. In the patient group that received OPT with the culprit drug, 12 out of 87 patients tested positive, corre-

Fig. 2 Hypersensitivity reactions to culprit drugs according to the patient's medical history. The frequency of hypersensitivity reactions to non-steroidal anti-inflammatory drugs in the medical history of 398 patients was assessed between 2012 and 2016. The $x$-axis shows the name of the culprit drugs, while the $y$-axis shows the frequency of hypersensitivity reactions

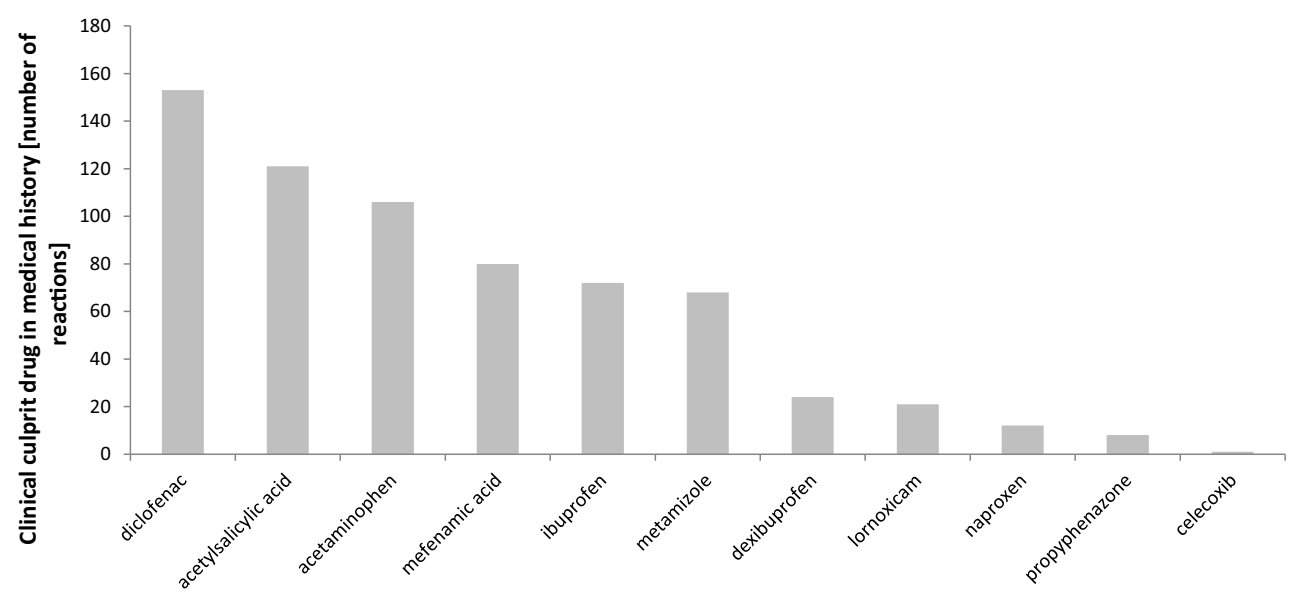


Table 2 Numbers of negative and positive skin test results for the culprit drugs and alternative non-steroidal antiinflammatory drugs (NSAIDs)

\begin{tabular}{l|l|l|l|}
\hline Skin test & Total & Alternative NSAIDs & Culprit drug \\
\hline Tested total & $361(90.7 \%)$ & - & - \\
\hline Tested positive & - & $41(10.3 \%)$ & $25(6.3 \%)$ \\
\hline
\end{tabular}

Table 3 Total numbers of positive reactions in skin tests and to the culprit drugs specifically

\begin{tabular}{|l|l|l|}
\hline Skin test & Positive in total & Positive to culprit drug \\
\hline Diclofenac & 19 & 8 \\
\hline Ibuprofen & 1 & 1 \\
\hline Propyphenazone & 20 & 3 \\
\hline Acetylsalicylic acid & 3 & 2 \\
\hline Metamizol & 19 & 8 \\
\hline Acetaminophen & 4 & 3 \\
\hline Total & 66 & 25 \\
\hline
\end{tabular}

sponding to a reaction rate of $13.79 \%$ (Table 4 ). The highest reaction rate to the culprit drug was observed for lornoxicam, which was administered in 10 patients leading to a positive reaction in six cases $(60.0 \%)$, followed by ibuprofen (40.0\%) and naproxen (33.3\%) (Table 4). Interestingly, diclofenac was responsible for most reactions in patients' medical history, but no reaction occurred during OPT with this culprit drug (Table 4).

In terms of OPTs with alternative NSAIDs, the tested NSAIDs were chosen individually, depending on the severity of the previous reaction and the specific NSAID (absolute/relative) COX2 inhibition (e.g. celecoxib, Iornoxicam), the lower upper provocation dose with a weaker NSAID (e.g. mefenamic acid, acetaminophen). In this setting, diclofenac caused the most reactions, followed by metamizole (13.33\%) and lornoxicam $(11.46 \%)$ as shown in Table 5 . The three most commonly tested drugs were lornoxicam (192 OPTs), acetaminophen (156 OPTs) and celecoxib (133 OPTs) with tolerability rates of $88.54 \%$ (hypersensitivity reactions $11.46 \%$ ), $92.31 \%$ (hypersensitivity reactions $7.69 \%$ ) and $91.73 \%$ (hypersensitivity reactions $8.27 \%$ ), respectively. Interestingly, patients had no reactions to meloxicam, mefenamic acid and in-
Table 4 Patients' medical history to the culprit drugs, the number of patients that received oral provocation tests (OPTs) with the culprit drug and the number of positive and negative reactions in OPTs with the culprit drugs

Table 5 Total numbers of oral provocation tests (OPTs) with alternative nonsteroidal anti-inflammatory drugs and the number of positive and negative reactions during OPTs

\begin{tabular}{|l}
\hline Tested drugs during OPT \\
Lornoxicam \\
\hline Ibuprofen \\
\hline Naproxen \\
\hline Acetylsalicylic acid \\
\hline Acetaminophen \\
\hline Diclofenac \\
\hline Mefenamic acid \\
\hline Metamizol \\
\hline Propyphenazone \\
\hline Meloxicam \\
\hline Indometacin \\
\hline Celecoxib \\
\hline Total \\
\hline
\end{tabular}

\begin{tabular}{|l|l|}
\hline Medical history \\
\hline 21 \\
96 \\
12 \\
\hline 121 \\
\hline 106 \\
\hline 153 \\
\hline 80 \\
\hline 68 \\
\hline 8 \\
\hline 1 \\
\hline 1 \\
\hline 1 \\
\hline 668 \\
\hline
\end{tabular}

\begin{tabular}{|l|l|l|l|}
\hline Total & Negative & Positive & \% Positive \\
\hline 10 & 4 & 6 & 60.0 \\
\hline 5 & 3 & 2 & 40.0 \\
\hline 3 & 2 & 1 & 33.3 \\
\hline 10 & 9 & 1 & 10.0 \\
\hline 49 & 47 & 2 & 4.08 \\
\hline 7 & 7 & 0 & 0.0 \\
\hline 1 & 1 & 0 & 0.0 \\
\hline 2 & 2 & 0 & 0.0 \\
\hline 0 & 0 & 0 & 0.0 \\
\hline 0 & 0 & 0 & 0.0 \\
\hline 0 & 0 & 0 & 0.0 \\
\hline 0 & 0 & 0 & 0.0 \\
\hline 87 & 75 & 12 & 13.79 \\
\hline
\end{tabular}

\begin{tabular}{|c|c|c|c|c|}
\hline Tested drugs during OPT & Total & Negative & Positive & $\%$ Positive \\
\hline Diclofenac & 16 & 13 & 3 & 18.75 \\
\hline Metamizole & 15 & 13 & 2 & 13.33 \\
\hline Lornoxicam & 192 & 170 & 22 & 11.46 \\
\hline Acetylsalicylic acid & 31 & 28 & 3 & 9.68 \\
\hline Placebo & 69 & 63 & 6 & 8.70 \\
\hline Celecoxib & 133 & 122 & 11 & 8.27 \\
\hline Acetaminophen & 156 & 144 & 12 & 7.69 \\
\hline Naproxen & 26 & 25 & 1 & 3.85 \\
\hline Ibuprofen & 82 & 80 & 2 & 2.44 \\
\hline Indometacin & 1 & 1 & 0 & 0.0 \\
\hline Mefenamic acid & 8 & 8 & 0 & 0.0 \\
\hline Meloxicam & 18 & 18 & 0 & 0.0 \\
\hline Total & 739 & 677 & 62 & 8.39 \\
\hline
\end{tabular}


domethacin in OPTs, demonstrating the favorable tolerability of these drugs in patients with NSAID hypersensitivity (Table 5). In addition, six out of 342 patients reacted to placebo (generalized pruritus, dyspnea, cough, flush in the face), which indicates the importance of placebo-controlled OPTs when testing for NSAID hypersensitivity.

\section{Discussion}

The present study showed that: (i) diclofenac is the most common drug with hypersensitivity reactions in the medical history of the current patient cohort and (ii) acetaminophen is a frequently used NSAID in the authors' geographical region with a favourable tolerability profile in OPT.

Based on medical history, diclofenac was the most common culprit drug with $153(38.4 \%)$ reactions. This is in contrast to other studies in which most of the reactions were caused by ASA [21, 22], ASA and ibuprofen [23], metamizole [11, 24] or arylpropionic acid derivatives [11]. ASA was found to be the second most common culprit drug according to medical history ( $n=121,30.4 \%)$ in the present patient cohort. In line with other reports, the rate of positive reactions to the culprit drug during OPT was $13.79 \%$, which is similar to observations by Schubert et al. [25] (13.8\% positive reactions) and Viola et al. [26] (22.2\% positive reactions). As ASA is the only irreversible blocker of COX-1, the low number of ASA provocation tests may be the reason for the low rate of positive provocation reaction tests. In contrast, studies in Turkish [24], Norwegian [27] and Latin American [28] cohorts found higher rates of positive reactions of respectively $25 \%, 32 \%$ and $52.3 \%$.

In the authors' patient cohort, $18.75 \%(n=3)$ had a positive reaction to diclofenac in OPT with alternative drugs. Picaud et al. [29] reported nine cases of anaphylaxis to diclofenac out of 33 patients in a French cohort for the period 2002-2012. This lower rate could be attributed to local differences in NSAID use. Furthermore, Picaud et al. [29] showed that an IgE-dependent mechanism might be the underlying cause of reactions to diclofenac and that the measurement of specific IgEs to diclofenac might be beneficial to diagnose diclofenac hypersensitivity. In the present cohort, all 24 patients that reacted to diclofenac during OPT had previous negative ST. These data are in line with Pérez-Alzate et al. [11], Gala et al. [30] and del Pozo et al. [31], showing a negative ST before oral challenge with diclofenac. The low rate of verified hypersensitivity reactions to diclofenac in the present study, which often avoided testing with the culprit drug, might be the result of OPT with alternative NSAIDs. This strategy helps to identify NSAIDs with a favorable tolerability profile and reduces the risk of severe hypersensitivity reactions during OPT.

A total of approximately $2 \%$ of the tested patients displayed objective symptoms to placebo in the present study. Interestingly, there are only scant data on placebo testing in NSAID hypersensitivity $[11,21,23]$. To identify unspecific reactions and accurately assess the rate of positive reactions to the tested drugs, it is necessary to also include those results in the overall assessment.

Unspecific reactions such as headache, localized pruritus and dizziness during OPT were the common reactions observed in the current study (53.8\%). These unspecific reactions have not been mentioned in other publications [11, 21, 23]. The authors' observations highlight the importance of placebo-controlled drug challenge designs to distinguish unspecific reactions/symptoms from hypersensitivity.

A limitation of the study is its retrospective design. In addition, delayed reactions and possible genetic factors for NSAID hypersensitivity $[11,24]$ or the correlation with chronic diseases (e.g. atopic dermatitis) were not included in the data analysis [24]. In this context, previous trials have shown that atopic dermatitis does not seem to influence hypersensitivity reactions to NSAIDs [1, 16, 32].

Taken together, oral provocation tests are the gold standard to identify safe and tolerable drugs for patients with a clinical history of hypersensitivity to NSAIDs. Skin testing is highly recommended in the management of NSAID intolerance and should be performed on a routine basis before OPT. The pharmacological agents used in OPT with NSAIDs should be individually chosen based on clinical history and ST, but also depending on their common use in different geographic regions.

Acknowledgements The authors kindly thank the patients who participated in this study.

Author Contribution W. Hoetzenecker, I. Angelova-Fischer and E. Guenova conceptualized and designed the study. T. Bangerl, B. Zahel and A. Lueger conducted the trial. T. Bangerl performed the statistical analyses. W. Hoetzenecker and I. Angelova-Fischer wrote the manuscript. All authors approved the final manuscript for submission.

Funding Open access funding provided by Johannes Kepler University Linz.

\section{Compliance with ethical guidelines}

Conflict of interest T. Bangerl, B. Zahel, A. Lueger, E. Guenova, I. Angelova-Fischer and W. Hoetzenecker declare that they have no competing interests.

Ethical standards All procedures performed in studies involving human participants were in accordance with the ethical standards of the institutional and/or national research committees and with the 1964 Declaration of Helsinki, as revised in 2013. Informed consent was obtained from all individual participants included in the study.

Open Access This article is licensed under a Creative Commons Attribution 4.0 International License, which permits use, sharing, adaptation, distribution and reproduction in any medium or format, as long as you give appropriate credit to the original author(s) and the source, provide a link to 
the Creative Commons licence, and indicate if changes were made. The images or other third party material in this article are included in the article's Creative Commons licence, unless indicated otherwise in a credit line to the material. If material is not included in the article's Creative Commons licence and your intended use is not permitted by statutory regulation or exceeds the permitted use, you will need to obtain permission directly from the copyright holder. To view a copy of this licence, visit http://creativecommons.org/licenses/by/4.0/.

\section{References}

1. GomesER, DemolyP.Epidemiology ofhypersensitivitydrug reactions. Curr Opin Allergy Clin Immunol. 2005;5:309-16.

2. GomesE, Cardoso MF, PraçaF, Gomes L, MariñoE, DemolyP. Self-reported drug allergy in a general adult Portuguese population. Clin ExpAllergy. 2004;34:1597-601.

3. Settipane RA, Constattine HP, Settipane GA. Aspirin intolerance and recurrent urticaria in normal adults and children: epidemiology and review. Allergy. 1980;35:149-54.

4. Pfaar O, Klimek L. Aspirin desensitization in aspirin intolerance: update on current standards and recent improvements. Curr Opin Allergy Clin Immunol. 2006;6:161-6.

5. Erbagci Z. Multiple NSAID intolerance in chronic idiopathic urticaria is correlated with delayed, pronounced and prolonged autoreactivity. J Dermatol. 2004;31:376-82.

6. Kim JE, Kountakis SE. The prevalence of Samter's triad in patients undergoing functional endoscopic sinus surgery. Ear Nose Throat J. 2007;86:396-9.

7. Kowalski ML, Agache I, Bavbek S, Bakirtas A, Blanca M, Bochenek G, et al. Diagnosis and management of NSAIDexacerbated respiratory disease (N-ERD) - a EAACI position paper. Allergy. 2019;74:28-39.

8. Canto MG, Andreu I, Fernandez J, Blanca M. Selective immediatehypersensitivity reactions to NSAIDs. Curr Opin Allergy Clin Immunol. 2009;9:293-7.

9. Quiralte J, Blanco C, Castillo R, Ortega N, Carrillo T. Anaphylactoid reactions due to nonsteroidal antiinflammatory drugs: clinical and cross-reactivity studies. Ann Allergy Asthma Immunol. 1997;78:293-6.

10. Asero R. Oral aspirin challenges in patients with a history of intolerance to single non-steroidal anti-inflammatory drugs. Clin ExpAllergy. 2005;35:713-6.

11. Pérez-Alzate D, Cornejo-García JA, Pérez-Sánchez N, Andreu I, García-Moral A, Agúndez JA, et al. Immediate reactions to more than 1 NSAID must not be considered cross-hypersensitivity unless tolerance to asa is verified. J InvestigAllergol Clin Immunol. 2017;27:32-9.

12. Wöhrl S. NSAID hypersensitivity-recommendations for diagnostic work up and patient management. Allergo J Int. 2018;27:114-21.

13. Laidlaw TM, Cahill KN. Current knowledge and management of hypersensitivity to aspirin and NSAIDs. J Allergy Clin Immunol Pract. 2017;5:537-45.

14. Nizankowska-Mogilnicka E, Bochenek G, Mastalerz L, ŚwierczyńskaM,PicadoC,ScaddingG, etal. EAACI/GA2LEN guideline: aspirin provocation tests for diagnosis of aspirin hypersensitivity. Allergy. 2007;62:1111-8.

15. Mirakian R, Ewan PW, Durham SR, Youlten LJF, Dugué P, Friedmann PS, et al. BSACI guidelines for the management of drug allergy. Clin Exp Allergy. 2009;39:43-61.

16. AbererW, Bircher A, Romano A, Blanca M, Campi P, Fernandez J, et al. Drug provocation testing in the diagnosis of drug hypersensitivity reactions: general considerations. Allergy. 2003;58:854-63.

17. Messaad D, Sahla H, Benahmed S, Godard P, Bousquet J, Demoly P. Drug provocation tests in patients with a history suggesting an immediate drug hypersensitivity reaction. Ann Intern Med. 2004;140:1001-6.

18. Ruëff F, Bergmann KC, Brockow K, Fuchs T, Grübl A, Jung K, et al. Hauttests zur Diagnostik von allergischen Soforttyp-Reaktionen: Leitlinie der Deutschen Gesellschaft für Allergologie und klinischen Immunologie (DGAKI). Pneumologie. 2011;65:484-95.

19. Kidon M, Blanca-Lopez N, Gomes E, Terreehorst I, Tanno L, Ponvert C, et al. EAACI/ENDA position paper: diagnosis and management of hypersensitivity reactions to nonsteroidal anti-inflammatory drugs (NSAIDs) in children and adolescents. Pediatr Allergy Immunol. 2018;29: 469-80.

20. Mueller HL. Diagnosis and treatment of insect sensitivity. JAsthma Res. 1966;3:331-3.

21. Gomes ER, Geraldes L, Gaspar Â, Malheiro D, Cadinha S, Abreu C, et al. Hypersensitivity reactions to nonsteroidal anti-inflammatory drugs among adults: clinical features and risk factors for diagnosis confirmation. Int Arch Allergy Immunol. 2017;171:269-75.

22. Quiralte J, Blanco C, Castillo R, Delgado J, Carrillo T. Intolerance to nonsteroidal antiinflammatory drugs: results of controlled drug challenges in 98 patients. J Allergy Clin Immunol. 1996;98:678-85.

23. Nissen CV, Bindslev-Jensen C, Mortz CG. Hypersensitivity to non-steroidal anti-inflammatory drugs (NSAIDs): classification of a danish patient cohort according to EAACI/ ENDA guidelines. Clin Transl Allergy. 2015;5:10.

24. Demir S, Olgac M, UnalD, GelincikA, Colakoglu B, Buyukozturk S. Evaluation of hypersensitivity reactions to nonsteroidal anti-inflammatory drugs according to the latest classification. Allergy. 2015;70:1461-7.

25. Schubert B, Grosse Perdekamp MT, Pfeuffer P, Raith P, Bröcker E-B, Trautmann A. Nonsteroidal anti-inflammatory drug hypersensitivity: fable or reality? Eur J Dermatol. 2005;15:164-7.

26. Viola M, Rumi G, Valluzzi RL, Gaeta F, Caruso C, Romano A. Assessing potential determinants of positive provocation tests in subjects with NSAID hypersensitivity. Clin Exp Allergy. 2011;41:96-103.

27. Chalabianloo F, Berstad A, Schjøtt J, Riedel B, Irgens A, Florvaag E. Clinical characteristics of patients with drug hypersensitivity in Norway: a single-centre study. Pharmacoepidemiol Drug Saf. 2011;20:506-13.

28. Jares EJ, Sánchez-Borges M, Cardona-Villa R, Ensina LF, Arias-Cruz A, Gómez M, et al. Multinational experience with hypersensitivity drug reactions in Latin America. Ann Allergy Asthma Immunol. 2014;113:282-9.

29. Picaud J, Beaudouin E, Renaudin JM, Pirson F, Metz-FavreC, Dron-Gonzalvez M, et al. Anaphylaxis to diclofenac: nine cases reported to the allergy vigilance network in France. Allergy. 2014;69:1420-3.

30. Gala G, Blanco R, Quirce S, Pérez-Camo I, Alvarez-Fernández JA, Díez-Gómez ML. Diclofenac-induced urticaria with aspirin tolerance. Allergy. 1998;53:623-4.

31. del Pozo MD, Lobera T, Blasco A. Selective hypersensitivity to diclofenac. Allergy. 2000;55:412-3.

32. Sánchez-Borges M, Capriles-Hulett A. Atopy is a risk factor for non-steroidal anti-inflammatory drug sensitivity. Ann Allergy Asthma Immunol. 2000;84:101-6. 